\title{
Mechanical sensitivity of the human
}

\section{conjunctiva}

\author{
Maria Navascues-Cornago BSc MSc
}

Carole Maldonado-Codina BSc MSc PhD

\section{Philip B Morgan BSc PhD}

Eurolens Research, Faculty of Life Sciences, The University of Manchester, Manchester, United Kingdom.

Corresponding author: Maria Navascues-Cornago, Eurolens Research, Carys Bannister building, The University of Manchester, Dover St, Manchester, M13 9PL.

e-mail: maria.navascuescornago@manchester.ac.uk, Tel: +44 1613060470

There is no conflict of interest to disclose.

This work forms part of a self-funded PhD programme of research.

Key words: ocular surface, conjunctiva, sensitivity, Cochet-Bonnet aesthesiometer, eyelid margin

THIS IS A NON-FINAL VERSION OF AN ARTICLE PUBLISHED IN FINAL FORM:

Navascues-Cornago M, Maldonado-Codina C \& Morgan (2014). Mechanical sensitivity of the human conjunctiva. Cornea, 33, 855-859 


\section{Abstract}

Purpose. To map the sensitivity of the marginal and other conjunctival regions and to investigate changes in the sensitivity of these regions between morning and evening.

Methods. Thirty-five healthy, non-contact lens wearers (20 female, 15 male; age $27.7 \pm$ 7.3 years) were enrolled. Mechanical sensitivity was measured at eight locations on the ocular surface and adnexa (cornea, marginal, bulbar and tarsal conjunctiva) using a Cochet-Bonnet aesthesiometer ( $0.12 \mathrm{~mm}$ diameter filament). A sub-group of 11 subjects ( 6 female, 5 male; age $28.9 \pm 9.9$ years) returned after $12 \mathrm{~h}$ when this protocol was repeated.

Results. The cornea was found to be the most sensitive region (all $p<0.001$ ). The marginal conjunctiva showed greater sensitivity than the bulbar and tarsal conjunctiva (all $p<0.001$ ). The temporal marginal conjunctiva was more sensitive than the central marginal conjunctiva (all $p<0.05$ ). No difference in marginal conjunctival sensitivity was found between upper and lower eyelids (all $p>0.05$ ). The upper tarsal conjunctiva was more sensitive than the lower tarsal conjunctiva $(p=0.04)$. There was no significant difference in sensitivity between the morning and the evening for any of the locations investigated (Bonferroni adjusted $p>0.006$ ).

Conclusion. This work has demonstrated that the marginal conjunctiva was the most sensitive of all the conjunctival regions and that this does not alter over the course of the day. 


\section{Introduction}

The sensitivity of the ocular surface is thought to play an important role in the comfort of contact lenses. ${ }^{1}$ In turn, contact lens discomfort (CLD) is important because it is the main reason for discontinuation ${ }^{2-4}$ of contact lens wear which in turn affects the growth of the contact lens industry. ${ }^{5}$

Recently, a number of publications have focused on characterising the lid margins and understanding how they interact with a contact lens. ${ }^{6-13}$ Intuitively, the lid margin is likely to play an important role in contact lens comfort because of its interaction with the lens surface. According to recent histological investigations, the lid margin has three distinct zones with clear anatomical differences. The keratinized epithelium of the epidermis extends over the meibomian gland orifices, and is here replaced by a layer of parakeratinized cells that represents the mucocutaneous junction. ${ }^{6}$ Following this transition zone, a conjunctival epithelium begins and forms an elevation at the peak of the inner lid border that gradually thinners towards the subtarsal fold. This epithelial thickening, known as the 'lid wiper', rubs against the ocular surface (or lens surface) during blinking and may be responsible for the spread of the tear film. ${ }^{6,7}$ Recent work by Papas et al ${ }^{14}$ has shown that end of day comfort is not influenced by lens replacement mid-way through a wearing day and lends weight to the hypothesis that the lens itself is inducing a 'fatigue-like' response in the ocular tissues.

Few studies have attempted to characterise the sensitivity of the lid margins themselves. ${ }^{15-18}$ Furthermore, unlike the diurnal variation in the sensitivity of the cornea ${ }^{19-21}$ and the bulbar conjunctiva, ${ }^{21}$ it is unknown whether the distinct subzones of the lid margin undergo such diurnal changes or indeed if these regions have different sensitivities. An improved understanding of the sensitivity of the conjunctiva (and in 
particular, the marginal conjunctiva) may ultimately contribute to a better understanding of the interaction between the ocular surface and the lid margin as well as a contact lens surface and the lid margin.

The purpose of this work was to map the sensitivity of the marginal conjunctiva of the upper and lower eyelids and other conjunctival regions in a group of normal, non-contact lens wearing subjects and to investigate whether the sensitivity of these areas changed over a 12 hour period. 


\section{Materials and methods}

\section{Study design}

This study was randomised, controlled, and subject-masked. Ethical approval for carrying out this clinical work was obtained from the University of Manchester Committee on the Ethics of Research on Human Beings. The study was conducted in accordance to the tenets of the Declaration of Helsinki. All the subjects were given information about the study before they signed a consent form to participate.

\section{Subjects}

To determine the study sample size, power analysis was employed using data from a previous pilot investigation. For differences in sensitivity between various ocular surface locations, it was demonstrated that if 33 subjects completed the morning visit, the power of the study to detect a $1 \mathrm{~cm}$ difference in sensitivity using a Cochet-Bonnet aesthesiometer (assuming a alpha of 0.05 , a two-tailed paired analysis and a standard deviation of differences of 2.0 ) is 0.80 . To allow for subject discontinuations, 35 subjects were recruited for the morning visit. For diurnal variation of sensitivity, it was calculated that if 10 subjects attended the evening visit, the power of the study to detect a $1 \mathrm{~cm}$ difference in sensitivity (assuming al alpha of 0.05 , a two-tailed paired analysis and a standard deviation of differences of 1.0$)$ is 0.80 .

\section{Experimental protocol}

Clinical measurements of both eyes were first taken in the morning at least 2 hours after awakening in order to avoid any possible effects from oedema. Details of the ocular history were recorded and an anterior eye examination was undertaken to ensure that all subjects met the inclusion and exclusion criteria outlined in Table 1. 
Mechanical sensitivity thresholds of the ocular surface were measured using a CochetBonnet aesthesiometer (Luneau Ophthalmologie, Paris, France) with a $0.12 \mathrm{~mm}$ diameter nylon filament. The Cochet-Bonnet aesthesiometer was mounted on a slit lamp to allow accurate application of the stimulus with the aid of the magnification observation system and also allowed the aesthesiometer to be controlled in the $\mathrm{x}, \mathrm{y}$ and $\mathrm{z}$ planes. This set-up also had the benefit of ensuring that the filament approached the ocular surface smoothly and perpendicularly. Mechanical thresholds were determined using the ascending method of limits. The filament at its longest was applied first and subjects were asked to indicate if they could feel the stimulus. If the subject could not feel the stimulus, the length of the filament was progressively reduced in $5 \mathrm{~mm}$ steps until the subject felt it. The first visible bending of the filament was used as an objective indicator of contact. Four stimuli were presented at each level and the mechanical threshold was determined by the filament length that gave two or more positive responses to the stimulus application.

The length of the filament could be converted into pressure units using the manufacturer's chart. However this conversion is not always accurate because factors such as aging of the nylon thread and ambient humidity can affect the filament. 22,23 Therefore, a calibration of the Cochet-Bonnet aesthesiometer was carried out in order to convert values of filament length into force exerted. The force exerted by the filament was measured with the instrument positioned vertically above a highly sensitive load cell (LSB200 S-Beam Jr. Load Cell, Futek Inc., Irvine, CA, U.S.A.). The filament was then applied until the first visible bending and the mean of five readings was plotted against the filament length (Figure 1). 
Mechanical thresholds were determined at the following locations (Figure 2): central cornea; temporal bulbar conjunctiva (approximately $3 \mathrm{~mm}$ from the limbus); upper and lower marginal conjunctiva (central and temporal, approximately $5 \mathrm{~mm}$ from the centre); and upper and lower tarsal conjunctiva ( $2 \mathrm{~mm}$ posterior to the line of Marx). The order of assessment of each of these locations was randomised for each eye. To measure the sensitivity of the marginal and tarsal conjunctiva, the upper eyelid was completely everted and the lower eyelid partially everted. The exact location of the sensitivity measurement for the marginal conjunctiva depended on whether there was lissamine green staining of the lid margin. When the lid margin displayed an area of staining the sensitivity was measured in the middle of the staining (in a vertical direction). If only the line of Marx was stained, the sensitivity was measured approximately $0.1 \mathrm{~mm}$ posterior to the line of Marx. An eyepiece graticule (Eyepiece BM 10x, Haag-Streit AG, Koeniz, Switzerland) was used at 10x magnification to perform these measurements accurately.

After 12 hours, repeated measurements were undertaken in a subgroup of 11 subjects to assess whether there had been a change in sensitivity over the course of the day. All measurements were taken at room temperature of $22.8^{\circ} \mathrm{C}$ (range: $21.4-24.2^{\circ} \mathrm{C}$ ) and relative humidity of $48.1 \%$ (range: $42.8-53.3 \%$ ).

\section{Statistical analysis}

Only data for the right eye were included in the analysis. Statistical analysis was carried out using SPSS Statistic version 20 (IBM Corp., Armonk, NY, U.S.A.). The Saphiro-Wilk test showed that variables were not normally distributed and therefore, nonparametric analysis was carried out. The Friedman test was used to analyse sensitivity data at the various ocular locations. Any significant difference was further investigated using the Wilcoxon signed ranks test. Differences in sensitivity over time were also analysed using 
the Wilcoxon signed ranks test. A p-value of $<0.05$ was considered to be statistically significant. The significance level was adjusted using a Bonferroni correction when multiple comparisons were carried out in order to avoid Type I errors. 


\section{Results}

Thirty-five non-contact lens wearers (20 female and 15 men; mean age $27.7 \pm 7.3$ years, range 19-57 years) were enrolled in the study. Clinical measurements were taken in the morning for all subjects and repeated measurements after 12 hours were taken in a subgroup of 11 subjects ( 6 female and 5 men; mean age $28.9 \pm 9.9$ years, range 22-57 years).

\section{Mechanical sensitivity}

Mechanical thresholds are reported as force exerted $(\mathrm{mN})$ by the nylon filament (Figure 1). Table 2 shows mechanical thresholds for the various stimulus locations in the morning. There was a significant difference in sensitivity between the various ocular locations (Friedman, $p<0.001$ ). The cornea was found to be the most sensitive region (Wilcoxon signed rank, all $\mathrm{p}<0.001$ ). The marginal conjunctiva showed greater sensitivity than the bulbar and tarsal conjunctiva (Wilcoxon signed rank, all $p<0.001$ ). No difference in marginal conjunctival sensitivity was observed between the upper and lower eyelids (Wilcoxon signed rank, all $p>0.05$ ). The sensitivity of the temporal marginal conjunctiva was significantly greater than that of the central marginal conjunctiva (Wilcoxon signed rank, all $p<0.05$ ). The tarsal conjunctiva of the upper eyelid showed greater sensitivity than that of the lower eyelid (Wilcoxon signed rank, $p=$ 0.04). Mechanical thresholds measured in the evening are compared to those in the morning in Table 3. There was no significant difference in sensitivity between the morning and the evening for any of the locations (Wilcoxon signed rank, Bonferroni adjusted all $p>0.006)$. 


\section{Discussion}

A number of publications have attempted to characterize the anatomy of the lid margins. ${ }^{6-10}$ This recent interest in the lid margin is partly due to its potential role in contact lens comfort. ${ }^{11-13}$ The characterisation of the sensitivity of the marginal conjunctiva may contribute to better understanding the interaction between the ocular surface and the lid margin. This study aimed to map the sensitivity of the marginal conjunctiva and other conjunctival regions of the upper and lower eyelids in normal, noncontact lens wearing subjects and to investigate whether the sensitivity of these regions changes over the course of a day.

The present study found the central cornea to be the most sensitive region which is in agreement with previous investigations. ${ }^{15,21}$ This finding is expected considering the nerve density present in the cornea. ${ }^{24}$ The marginal conjunctiva showed greater sensitivity compared to other conjunctival regions. Previous studies have shown an increased sensitivity of the lid margins compared to other conjunctival surfaces such as the tarsal conjunctiva ${ }^{17}$ or bulbar conjunctiva. ${ }^{15}$ The greater sensitivity of the temporal marginal conjunctiva compared to the central marginal conjunctiva was unexpected considering that previous investigations have shown no difference between the central, temporal and nasal regions within the lid margin. ${ }^{15,16}$ There was no difference between upper and lower eyelid for the marginal conjunctival sensitivity. This finding agrees with a previous study, which recorded little difference between the upper and lower lid margins. ${ }^{15}$ However, other studies have shown contrary results, reporting a greater sensitivity for the lower lid margin compared to the upper eyelid. ${ }^{17,18}$ Taking into account that the lid margin is subdivided into three anatomically differentiated zones, ${ }^{6,7}$ the 
sensitivity of the lid margin is likely to vary depending on the stimulated zone. In the present study the sensitivity of the marginal conjunctiva was measured in the region displaying lissamine green staining, and thus the results may differ from the sensitivity of the lid margin assessed in other studies. The increased sensitivity of the marginal conjunctiva may suggest that it is important to consider not only the cornea but also the marginal conjunctiva as being an important determinant driving contact lens comfort. The sensory innervation of the human eyelid contains a complex variety of sensory nerve terminals. Light and electron microscopy studies have shown that both corpuscular (specialised) and free (non-specialised) nerve endings (FNEs) are commonly presented in the lid margin area, while only FNEs are observed in the tarsal conjunctiva. ${ }^{25}$ This sensory distribution may explain the greater sensitivity of the marginal conjunctiva compared to the tarsal conjunctiva found in the present study.

The tarsal conjunctiva of the upper eyelid was more sensitive than that of the lower eyelid. Variation in the number of nerve endings between the upper and lower eyelids could explain differences in sensitivity. ${ }^{25}$ However, regional variation in sensory innervation has not been fully investigated yet. In addition, it is important to consider that everting the upper eyelid prior to measuring the sensitivity may have affected the results.

The diurnal variation of the sensitivity of the various ocular regions was also investigated. This study showed no significant difference in sensitivity after a 12 hour period for any of the locations. Contrary to previous studies which showed that corneal sensitivity was lower in the morning compared to the evening, ${ }^{19-21}$ this study showed similar mechanical thresholds in the morning and the evening. Unlike corneal sensitivity, no diurnal variation has been previously reported for conjunctival sensitivity, ${ }^{21}$ and there are no previous reports investigating the diurnal variation of marginal conjunctival sensitivity. There are two facts that may explain the absence of difference for corneal 
thresholds. First, more pronounced changes in sensitivity may occur immediately after awakening. ${ }^{19,20}$ However, in this study, sensitivity measurements were taken at least 2 hours after awakening in order to avoid overnight corneal oedema which could affect the sensitivity measurements. ${ }^{20}$ Secondly, corneal thresholds in the morning were at the minimum intensity stimulus possible with the Cochet-Bonnet (i.e. at $6 \mathrm{~cm}$ filament length) in all the subgroup of 11 subjects. Therefore, if corneal sensitivity had increased towards the end of the day, it would not have been possible to detect this change as the thresholds would have been beyond the intensity range of the aesthesiometer. This finding highlights the main limitation of the Cochet-Bonnet instrument which is its truncated stimulus intensity range. The minimum intensity stimulus possible with the $0.12 \mathrm{~mm}$ diameter filament is suprathreshold for most subjects for the most sensitive ocular regions (e.g. the cornea), which results in an underestimation of corneal sensitivity. ${ }^{26,27}$ Murphy et al. $^{26}$ investigated this issue by comparing corneal sensitivity measured using two different instruments: the Cochet-Bonnet aesthesiometer and a Non-Contact Corneal Aesthesiometer (NCCA), which was able to measure at levels below the limits of the Cochet-Bonnet. They found that corneal thresholds measured using the Cochet-Bonnet were mainly clustered around the $6 \mathrm{~cm}$ filament length and the instrument was unable to measure corneal thresholds in $60.9 \%$ of subjects. Despite these apparent benefits of the NCCA, this device is unsuitable for work such as that carried out in the present investigation because the size and location of the stimulated area is poorly defined.$^{28}$ In the present study, corneal thresholds in the morning were at the minimum intensity stimulus possible with the Cochet-Bonnet in $82.9 \%$ of subjects. The limited intensity range delivered by the Cochet-Bonnet may have also affected the assessment of the diurnal variation of the marginal conjunctival sensitivity. Thresholds of three regions of the marginal conjunctiva (upper temporal, lower central and temporal locations) were at the measurable limit of the filament in between 45 and $63 \%$ of 
subjects. These limitations suggest that differences in sensitivity (either between locations or between time points) may not be detectable; however, those differences that are reported here to be statistically significant can be considered to be real.

In summary, the present study has shown that the marginal conjunctiva is the most sensitive of all the conjunctival regions. This work has also demonstrated for the first time that the sensitivity of the marginal conjunctiva does not change over the course of a day in normal, non-contact lens wearing subjects. This work provides further insight into the interaction between the marginal conjunctiva and the ocular surface. Future research should be conducted to characterize the sensitivity of the marginal conjunctiva in contact lens wearers in order to understand the interaction between the lid margin and the lens surface as well as the nature of CLD. 


\section{References}

1. Stapleton F, Marfurt C, Golebiowski B, et al. The TFOS International Workshop on Contact Lens Discomfort: Report of the Subcommittee on Neurobiology. Invest Ophthalmol Vis Sci. 2013;54:71-97.

2. Pritchard N, Fonn D, Brazeau D. Discontinuation of contact lens wear: a survey. Int Contact Lens Clin. 1999;26:157-62.

3. Richdale KODMS, Sinnott LTP, Skadahl EBS, et al. Frequency of and Factors Associated With Contact Lens Dissatisfaction and Discontinuation. Cornea. 2007;26:168-74.

4. Young G, Veys J, Pritchard N, et al. A multi-centre study of lapsed contact lens wearers. Ophthalmic Physiol Opt. 2002;22:516-27.

5. Fonn D. Preventing Contact Lens Dropouts. Addressing issues of discomfort, inconvenience and dryness may keep more patients in contact lens wear. Contact Lens Spectrum [serial online]. August 2002: Available from: http://www.clspectrum.com/articleviewer.aspx?articleid=12186. January 5, 2014.

6. Knop E, Knop N, Zhivov A, et al. The lid wiper and muco-cutaneous junction anatomy of the human eyelid margins: an in vivo confocal and histological study. $J$ Anat. 2011;218:449-61.

7. Knop N, Korb DR, Blackie CA, et al. The lid wiper contains goblet cells and goblet cell crypts for ocular surface lubrication during the blink. Cornea. 2012.

8. Jalbert I, Madigan MC, Shao M, et al. Assessing the human lid margin epithelium using impression cytology. Acta Ophthalmol. 2012;90:e547-e52.

9. Korb DR, Blackie CA. Meibomian gland diagnostic expressibility: Correlation with dry eye symptoms and gland location. Cornea. 2008;27:1142-7. 
10. Stahl UG, Delaveris A, Madigan M, et al. 9 Dallos Award Winner Lid wiper epitheliopathy: exploring the links to comfort and osmolality in contact lens wear. Cont Lens Anterior Eye. 2011;34, Supplement 1:S18.

11. Korb DR, Greiner JV, Herman JP, et al. Lid-wiper epitheliopathy and dry-eye symptoms in contact lens wearers. CLAO J. 2002;28:211-6.

12. Korb DR, Herman JP, Greiner JV, et al. Lid wiper epitheliopathy and dry eye symptoms. Eye Contact Lens. 2005;31:2-8.

13. Yeniad B, Beginoglu M, Bilgin LK. Lid-wiper epitheliopathy in contact lens users and patients with dry eye. Eye Contact Lens. 2010;36:140-3.

14. Papas EB, Tilia D, Tomlinson D, et al. Consequences of wear interruption for discomfort with contact lenses. Optom Vis Sci. 2013.

15. Norn MS. Conjunctival sensitivity in normal eyes. Acta Ophthalmol. 1973;51:58-66.

16. Lowther GE, Hill RM. Sensitivity threshold of the lower lid margin in the course of adaptation to contact lenses. Am J Optom Arch Am Acad Optom. 1968;45:587-94.

17. McGowan DP, Lawrenson JG, Ruskell GL. Touch sensitivity of the eyelid margin and palpebral conjunctiva. Acta Ophthalmol (Copenh). 1994;72:57-60.

18. Golebiowski B, Chim K, So J, et al. Lid margins: sensitivity, staining, meibomian gland dysfunction, and symptoms. Optom Vis Sci. 2012;89:e1443-9.

19. Millodot M. Diurnal variation of corneal sensitivity. Br J Ophthalmol. 1972;56:844-7.

20. Du Toit R, Vega JA, Fonn D, et al. Diurnal variation of corneal sensitivity and thickness. Cornea. 2003;22:205-9.

21. Golebiowski B, Papas EB, Stapleton F. Factors affecting corneal and conjunctival sensitivity measurement. Optom Vis Sci. 2008;85:E241-E6.

22. Brennan NA, Bruce AS. Esthesiometry as an indicator of corneal health. Optom Vis Sci. 1991;68:699-702. 
23. Murphy PJ, Patel S, Marshall J. A new non-contact corneal aesthesiometer (NCCA). Ophthalmic Physiol Opt. 1996;16:101-7.

24. Müller LJ, Marfurt CF, Kruse F, et al. Corneal nerves: structure, contents and function. Exp Eye Res. 2003;76:521-42.

25. Munger BL, Halata Z. The sensorineural apparatus of the human eyelid. Am J Anat. 1984;170:181-204.

26. Murphy PJ, Lawrenson JG, Patel S, et al. Reliability of the non-contact corneal aesthesiometer and its comparison with the Cochet-Bonnet aesthesiometer. Ophthalmic Physiol Opt. 1998;18:532-9.

27. Golebiowski B, Papas EB, Stapleton F. Assessing the sensory function of the ocular surface: Implications of use of a non-contact air jet aesthesiometer versus the Cochet-Bonnet aesthesiometer. Exp Eye Res. 2011;92:408-13.

28. Golebiowski B, Lim M, Papas E, et al. Understanding the stimulus of an air-jet aesthesiometer: computerised modelling and subjective interpretation. Ophthalmic Physiol Opt. 2013;33:104-13. 
Figure legends

Figure 1. Force exerted by the Cochet-Bonnet aesthesiometer at various filament lengths

Figure 2. Location of sensitivity measurements 


\section{Table 1. Inclusion and exclusion criteria}

\begin{tabular}{|l|l|}
\hline Inclusion criteria & \begin{tabular}{l} 
Of legal age (18 years) and capacity to volunteer \\
Understand their rights as a research subject \\
Willing and able to sign a Statement of Informed Consent \\
Willing and able to follow the protocol \\
Must never have worn contact lenses, i.e. must not currently be \\
wearing contact lenses and in the past must never have been \\
dispensed with contact lenses for more than two weeks \\
\hline Exclusion criteria
\end{tabular} \\
$\begin{array}{l}\text { Systemic or ocular disorder that may affect ocular health. } \\
\text { Grade } 2 \text { or greater of any anterior ocular clinical signs } \\
\text { Use of any topical medication such as eye drops or ointment } \\
\text { Previous cataract or corneal refractive surgery } \\
\text { Pregnant or lactating. }\end{array}$ \\
\hline
\end{tabular}


Table 2. Median (interquartile range) mechanical thresholds for the various stimulus locations $(\mathrm{N}=35)$.

\begin{tabular}{|l|c|}
\hline Stimulus location & Mechanical thresholds (mN) \\
\hline Cornea & $0.22(0.22-0.22)$ \\
\hline Bulbar conjunctiva & $1.19(0.89-1.84)$ \\
\hline Upper lid central marginal & $0.38(0.22-0.54)$ \\
\hline conjunctiva & $0.22(0.22-0.38)$ \\
\hline Upper lid temporal marginal & $1.84(0.70-3.57)$ \\
\hline conjunctiva & $0.38(0.30-0.43)$ \\
\hline cower lid central marginal & $0.89(0.54-1.84)$ \\
\hline Lower lid temporal marginal & $0.30(0.22-0.38)$ \\
\hline Upper lid tarsal conjunctiva & \\
\hline
\end{tabular}


Table 3. Comparison of median (interquartile range) mechanical thresholds in the morning and the evening.

\begin{tabular}{|c|c|c|c|c|}
\hline Location & $\begin{array}{c}\text { Number of } \\
\text { subjects }\end{array}$ & $\begin{array}{c}\text { Morning } \\
(\mathrm{mN})\end{array}$ & $\begin{array}{c}\text { Evening } \\
(\mathrm{mN})\end{array}$ & $P$-value \\
\hline Cornea & 11 & $\begin{array}{c}0.22(0.22- \\
0.22)\end{array}$ & $\begin{array}{c}0.22(0.22- \\
0.22)\end{array}$ & 1.00 \\
\hline Bulbar conjunctiva & 11 & $\begin{array}{c}0.89(0.70- \\
1.19)\end{array}$ & $\begin{array}{c}0.70(0.43- \\
1.84)\end{array}$ & 0.67 \\
\hline Upper lid central marginal conjunctiva & 11 & $\begin{array}{c}0.30(0.30- \\
0.38)\end{array}$ & $\begin{array}{c}0.22(0.22- \\
0.30)\end{array}$ & 0.01 \\
\hline Upper lid temporal marginal conjunctiva & 11 & $\begin{array}{c}0.22(0.22- \\
0.38)\end{array}$ & $\begin{array}{c}0.22(0.22- \\
0.30)\end{array}$ & 0.79 \\
\hline Lower lid central marginal conjunctiva & 11 & $\begin{array}{c}0.22(0.22- \\
0.38)\end{array}$ & $\begin{array}{c}0.22(0.22- \\
0.30)\end{array}$ & 0.75 \\
\hline Lower lid temporal marginal conjunctiva & 11 & $\begin{array}{c}0.30(0.22- \\
0.30)\end{array}$ & $\begin{array}{c}0.22(0.22- \\
0.22)\end{array}$ & 0.18 \\
\hline Upper lid tarsal conjunctiva & 11 & $\begin{array}{c}0.70(0.70- \\
1.19)\end{array}$ & $\begin{array}{c}0.54(0.38- \\
0.89)\end{array}$ & 0.07 \\
\hline Lower lid tarsal conjunctiva & 11 & $\begin{array}{c}0.89(0.70- \\
1.84)\end{array}$ & $\begin{array}{c}0.89(0.43- \\
8.69)\end{array}$ & 0.78 \\
\hline
\end{tabular}


Figure 1. Force exerted by the Cochet-Bonnet aesthesiometer at various filament lengths.

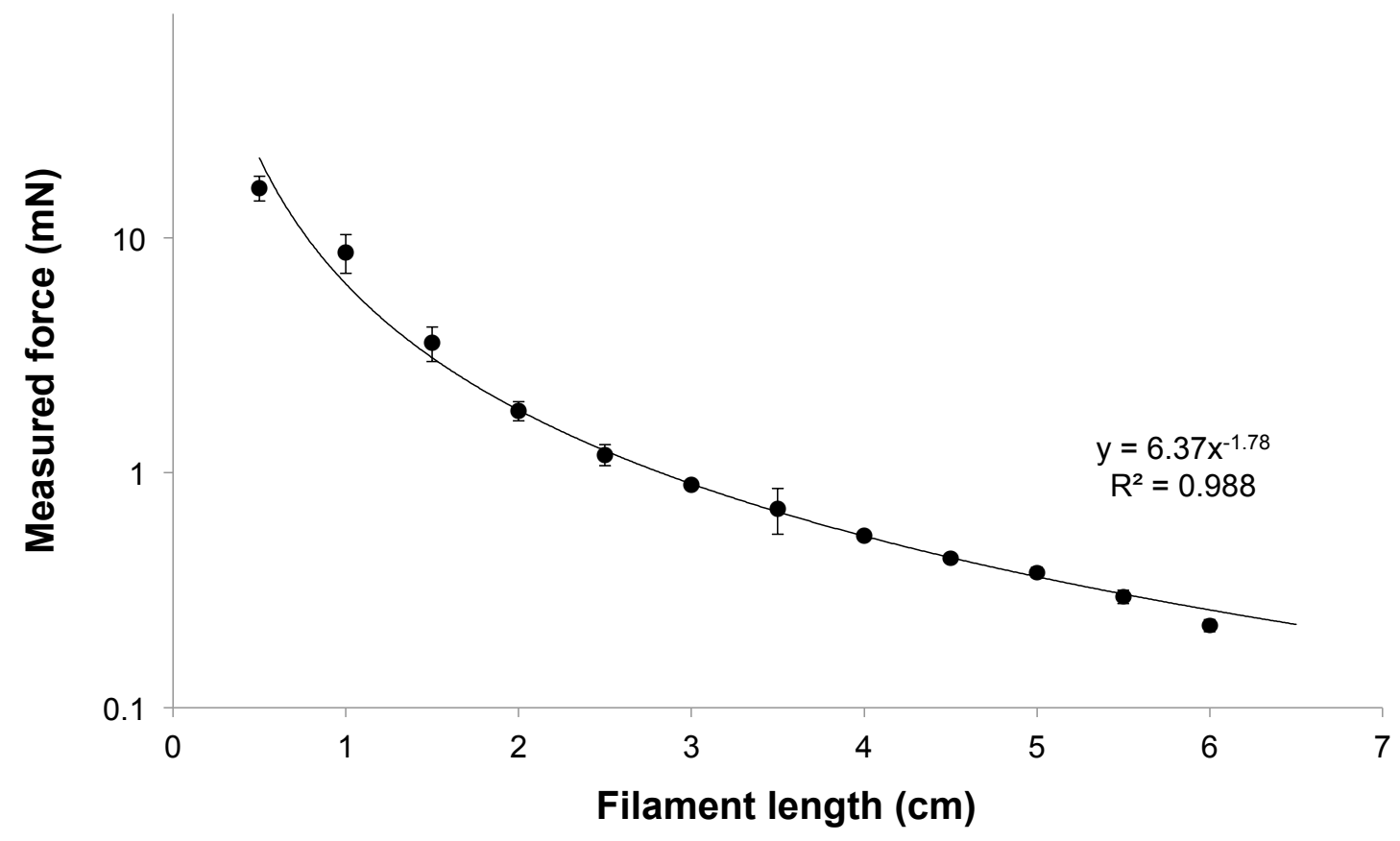


Figure 2. Location of the sensitivity measurements.
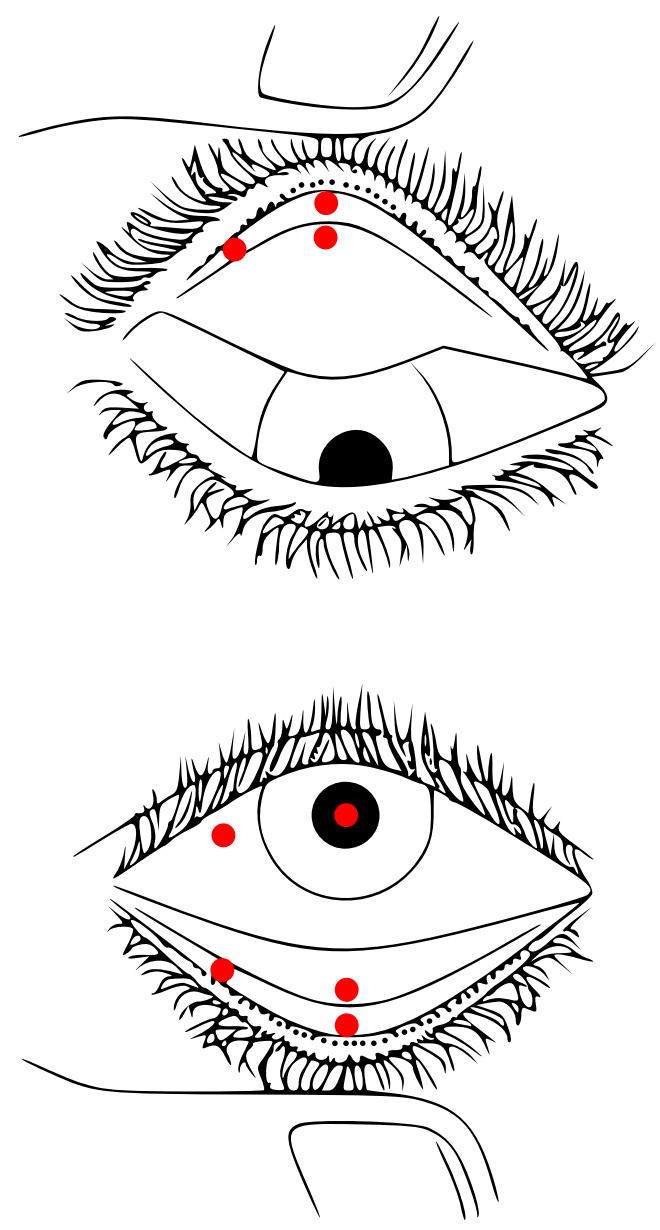
